OPEN ACCESS

Edited by:

Kulmira Nurgali,

Victoria University, Australia

Reviewed by:

Teodora Alexa-Stratulat,

Grigore T. Popa University of Medicine

and Pharmacy, Romania

Marcel Verheij,

Antoni van Leeuwenhoek Hospital,

Netherlands

*Correspondence:

Yefei Shu

shuyefei360423@163.com

Song Zheng

tztree@126.com

Specialty section:

This article was submitted to

Pharmacology of Anti-Cancer Drugs,

a section of the journal

Frontiers in Oncology

Received: 30 March 2021

Accepted: 14 June 2021

Published: 05 July 2021

Citation:

Shu Y and Zheng S (2021) Case

Report: Severe Rashes Associated

With Fruquintinib in a Patient With

Metastatic Colorectal Cancer.

Front. Oncol. 11:688231.

doi: 10.3389/fonc.2021.688231

\section{Case Report: Severe Rashes Associated With Fruquintinib in a Patient With Metastatic Colorectal Cancer}

\author{
Yefei Shu ${ }^{1 *}$ and Song Zheng ${ }^{1,2 *}$ \\ ${ }_{1}^{1}$ Department of Medical Oncology, Affiliated Hangzhou Cancer Hospital, Zhejiang University School of Medicine, Hangzhou, \\ China, ${ }^{2}$ Department of Medical Oncology, Affiliated Hangzhou First People's Hospital, Zhejiang University School of Medicine, \\ Hangzhou, China
}

Metastatic colorectal cancer $(\mathrm{mCRC})$ is a common and high-risk malignant tumor. Fruquintinib is a novel small-molecule compound with high selective inhibition of vascular endothelial growth factor (VEGF) receptor (VEGFR) for mCRC for which second-line or higher standard chemotherapy has been ineffective. A female patient with $\mathrm{mCRC}$ developed severe rashes after 2 weeks of taking fruquintinib. Considering the relationship between fruquintinib and the rashes, she discontinued taking the drug, and her condition improved. Although fruquintinib has shown good safety and manageable toxicity in previous trials, the patient in the present case developed severe rashes after 2 weeks of taking fruquintinib. The common skin reactions of hand and foot are erythema and paresthesia of hand and foot. Because few people have reported a severe rash caused by fruquintinib, which is different from the common hand foot skin reaction. We hope the case attracts the attention of oncologists.

Keywords: colorectal cancer, fruquintinib, rash, vascular endothelial growth factor receptor, case report

\section{INTRODUCTION}

Colorectal cancer ranks third and second in the incidence rate of male and female malignancies respectively all over the world (1). There are approximately 1.36 million new patients with colorectal cancer and nearly 700,000 related deaths every year, which is a huge global challenge. In China, the number of new cases is 376,000 every year, and this number continues to grow. In approximately $50 \%$ of all cases, colorectal cancer may eventually develop into mCRC or advanced colorectal cancer. After failure of second-line standard treatment, effective treatments of $\mathrm{mCRC}$ are limited, and some patients have good resilience and a strong desire for survival.

Fruquintinib is a novel small-molecule compound with high selective inhibition of vascular endothelial growth factor (VEGF) receptor (VEGFR) for mCRC for which second-line or higher standard chemotherapy has been ineffective (2). Although fruquintinib has shown the advantages of a strong effect, low toxicity, and good tolerance for colorectal cancer, it is associated with some unrecognized adverse reactions. 


\section{CASE REPORT}

A 71-year-old woman was admitted to hospital due to increased stool frequency and abdominal discomfort. She had no family history of cancer or drug allergies. She underwent radical resection of rectal cancer in May 2018. Postoperative pathology showed that rectal moderately differentiated adenocarcinoma was $3.5 \times 2.5 \mathrm{~cm}$ with negative margin. The depth of ulcer was full-thickness infiltration, involving serosa. One of nine lymph nodes had cancer metastasis. Special examination showed MSH2 (+), MSH6 (+), MLH1 (+), PMS2 (+), CDX2 (+). According to the Union for International Cancer Control/American Joint Committee on Cancer tumor-node-metastasis staging system (8th Edition, 2017), the postoperative pathological stage was stage IIIB (T3N1M0). The patient did not receive preoperative and postoperative radiotherapy. Eleven cycles of the FOLFOX6 regimen and one cycle of Xeloda were administered after the surgery, and the patient was then followed up regularly. In May 2019, intrahepatic nodules and peri-intestinal metastasis were considered. Positron emission tomography-computed tomography (CT) showed ascending-colon mesangial and omental nodules, liver S4 and S2 nodules, with increased fluorodeoxyglucose metabolism. Gene detection indicated she harbored wild-type KRAS/NRAS/BRAF, and microsatellite instability detection indicated microsatellite stability (MSS). Therefore, from May 16, 2019, FOLFIRI combined with cetuximab was administered for 12 courses, and from January 20, 2020, cetuximab (700 mg) was administered for two courses as maintenance treatment. When liver metastasis occurred, stereotactic body radiation therapy was performed in other hospital. The plan of radiotherapy is unknown. From May 19, 2020 , three courses of raltitrexed, oxaliplatin, bevacizumab (q2w) were administered. On July 21, 2020, pelvic and abdominal cavity CT was performed to evaluate disease progression. Although the patient was a MSS colorectal patient, based on the regonivo study (3) with ORR $36 \%$ and PFS 7.9 months and her strong desire for survival, she tried small molecule targeted drugs combined with immunotherapy. From July 24, 2020, regorafenib (80 $\mathrm{mg}$ QD) oral targeted therapy plus treprizumab $(240 \mathrm{mg})$ immunotherapy were administered. On September 30, 2020, because of fatigue, hand and foot skin reaction, and loss of appetite, the treatment plan was adjusted to TAS-102. Then, physical examination revealed a large amount of ascites. On December 17, 2020, the whole abdominal enhanced CT in our hospital showed liver II and V metastases considered, a large number of peritoneal effusion, and multiple metastases of omentum and mesentery (Figure 1). The disease progressed; therefore, the treatment was changed to fruquintinib. On January 12, 2021, the patient developed severe rashes after receiving fruquintinib ( $3 \mathrm{mg} \mathrm{qd}$ ) for approximately 2 weeks (Figures 2A, B). The patient developed severe rashes (Common Terminology Criteria for Adverse Events grade 3) in both lower limbs, which affected the patient's quality of life. Blood routine and coagulation function were basically normal. A dermatology consultation revealed dark purple infiltrative rashes scattered on both lower limbs and pustules observed locally. Betamethasone ( $1 \mathrm{ml}$, intramuscular injection), chlorphenamine maleate (10 mg, intramuscular injection), mupirocin ointment $(0.2 \mathrm{~g}$, bid for external use), and mometasone furoate cream (5 mg, QN for external use) were recommended. The dermatologist diagnosed these rashes as drug-induced rashes. Then the patient discontinued taking fruquintinib due to severe rashes. The severe rashes disappeared gradually after discontinuation of fruquintinib for 1 week. On January 22, 2021, the patient
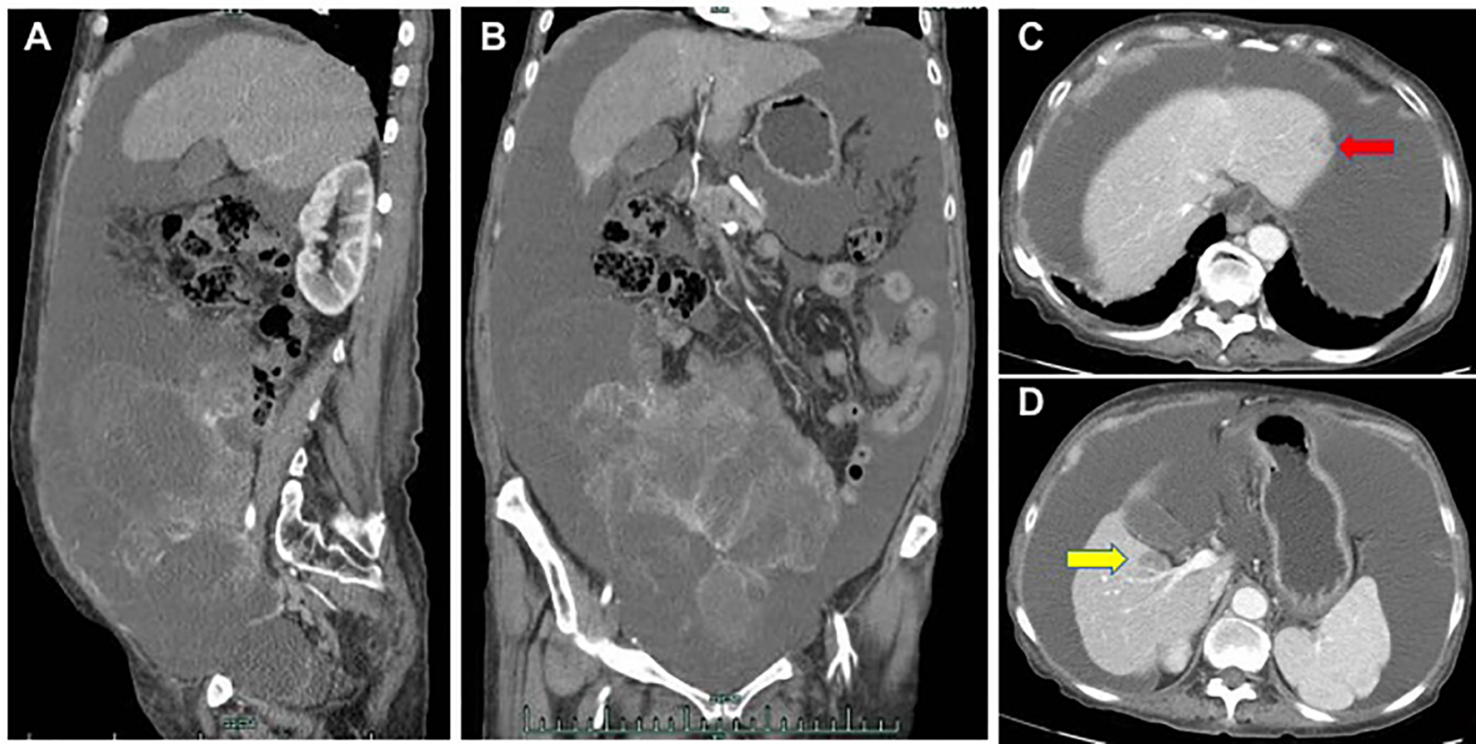

FIGURE 1 | On December 17, 2020, the whole abdominal enhanced CT in our hospital showed a large number of peritoneal effusion, multiple metastases of omentum and mesentery (A, B), liver II metastases (C, red arrow) and $\mathrm{V}$ metastases (D, yellow arrow). 
began to reduce the use of fruquintinib ( $1 \mathrm{mg}$ QD), and there was no recurrence of rashes. On March 2, 2021, the intraperitoneal tumor was found to have progressed; therefore, fruquintinib was discontinued. In the follow-up, the patient is receiving the best supportive treatment. The case timeline is presented in Figure 3.

Informed written consent was obtained from the patient prior to publication of this report and ethical approval was given by Hangzhou Cancer Hospital.

\section{DISCUSSION}

Fruquintinib is a novel small molecule compound with high selective inhibition of VEGFR1, -2, and, -3 (4). It inhibits VEGFinduced VEGFR2 phosphorylation, endothelial cell proliferation, and tubule formation (5). The approval of fruquintinib is based on a multicenter, randomized, double-blind, placebo-controlled phase III clinical study, Fresco. The results of the Fresco study showed that the median overall survival of patients with mCRC treated with fruquintinib was 9.3 months, which was 2.7 months longer than that in the placebo group; in addition, the median progression free survival of patients treated with fruquintinib was 3.7 months, which was significantly longer than 1.8 months in the placebo group, with a significant survival benefit (6).

However, in the Fresco study, grades 3 and 4 treatmentemergent adverse events occurred in $61.2 \%$ of patients who received fruquintinib. The most common adverse reactions affecting patients in the fruquintinib group were hypertension, proteinuria, hand foot skin reactions, etc., which were related to the target VEGFR, and the prevalence was relatively low, which was also controllable clinically, and most reactions were tolerable (7). Hand foot syndrome (palmar or plantar swelling, or pain or fingertip erythema) is the most common skin adverse reaction to fruquintinib, which is usually mild to moderate (grade 1-2). Fruquintinib also exhibits cross-toxicity with other anti-vascular drugs (such as bevacizumab), including hypertension, proteinuria, bleeding. A meta-analysis has shown that fruquintinib exhibits less toxicity among all-grade toxicities when compared with that of regorafenib (8).

The common skin reactions of hand and foot are erythema and paresthesia of hand and foot. Because few people have reported a severe rash caused by fruquintinib, which is different from the common hand foot skin reaction. The mechanism of fruquintinib induced rash is not very clear. We think it may be related to the inhibition of VEGFR2/3 phosphorylation by fruquintinib, thus inhibiting the proliferation and lumen formation of endothelial cells. The specific mechanism of severe skin rash caused by fruquintinib deserves further exploration.

\section{CONCLUSION}

Cancer therapy faces the challenge of handling a double-edged sword. Fruquintinib brings not only clinical benefits, but also some adverse reactions. How to manage fruquintinib to maximize the therapeutic effect and avoid adverse reactions as much as possible is a problem we need to explore.
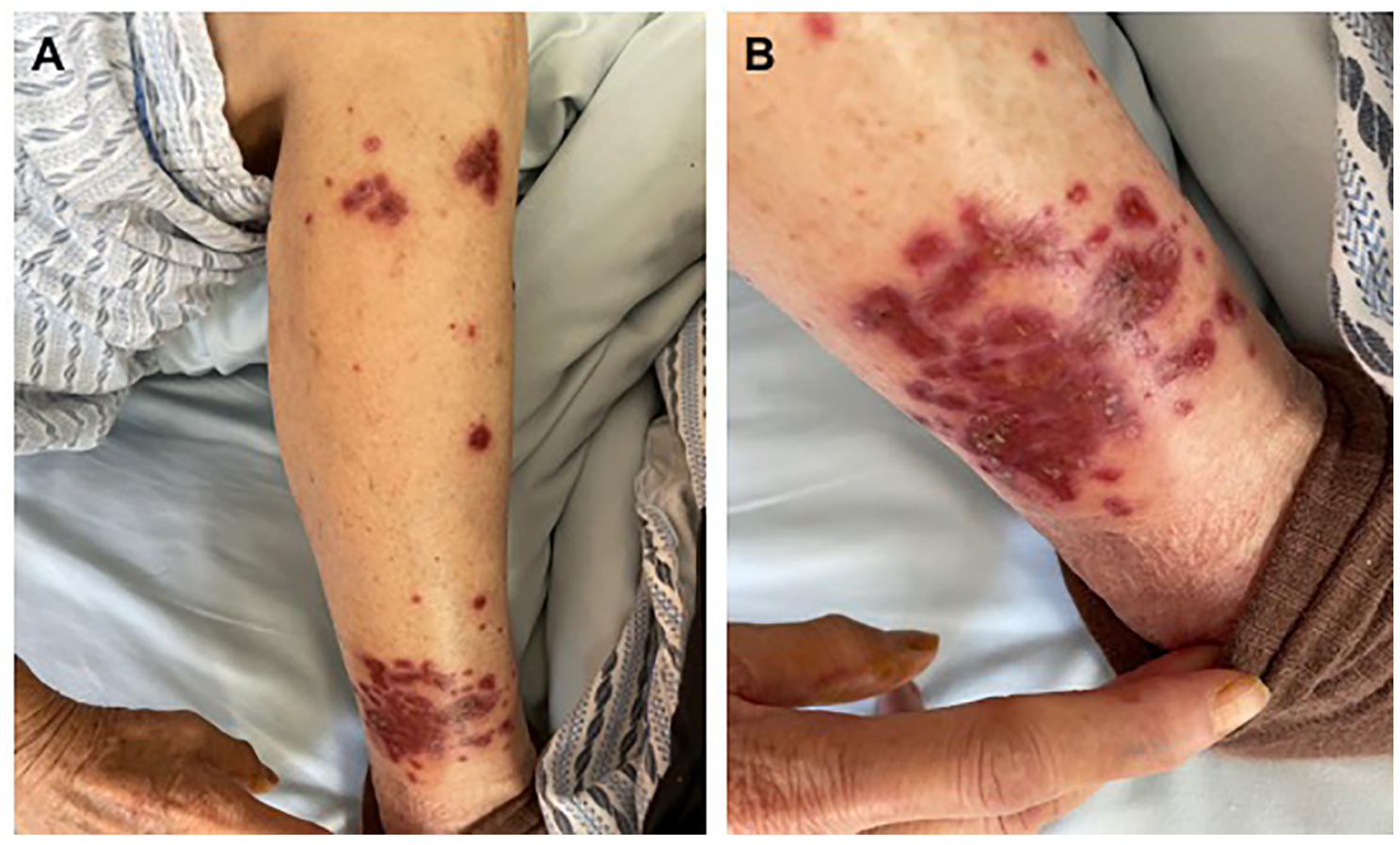

FIGURE 2 | (A, B) The severe rashes after receiving fruquintinib for approximately 2 weeks. 


- Radical resection of rectal cancer
May, 11 cycles of FOLFOX6 and 1 cycle of Xeloda
2018

FIGURE 3 | The case timeline.

\section{DATA AVAILABILITY STATEMENT}

The raw data supporting the conclusions of this article will be made available by the authors, without undue reservation.

\section{ETHICS STATEMENT}

The studies involving human participants were reviewed and approved by Hangzhou Cancer Hospital. Written informed

\section{REFERENCES}

1. GBD 2017 Colorectal Cancer Collaborators. The Global, Regional, and National Burden of Colorectal Cancer and Its Attributable Risk Factors in 195 Countries and Territories, 1990-2017: A Systematic Analysis for the Global Burden of Disease Study 2017. Lancet Gastroenterol Hepatol (2019) 4(12):91333. doi: 10.1016/S2468-1253(19)30345-0

2. Zhang Y, Zou JY, Wang Z, Wang Y. Fruquintinib: A Novel Antivascular Endothelial Growth Factor Receptor Tyrosine Kinase Inhibitor for the Treatment of Metastatic Colorectal Cancer. Cancer Manag Res (2019) 11:7787-03. doi: 10.2147/CMAR.S215533

3. Fukuoka S, Hara H, Takahashi N, Kojima T, Kawazoe A, Asayama M, et al. Regorafenib Plus Nivolumab in Patients With Advanced Gastric or Colorectal Cancer: An Open-Label, Dose-Escalation, and Dose-Expansion Phase Ib Trial (REGONIVO, EPOC1603). J Clin Oncol 38(18):2053-61. doi: 10.1200/ JCO.19.03296

4. Chen Z, Jiang L. The Clinical Application of Fruquintinib on Colorectal Cancer. Expert Rev Clin Pharmacol (2019) 12(8):713-21. doi: 10.1080/17512433.2019.1630272

5. Deng Y, Li X. Fruquintinib and its Use in the Treatment of Metastatic Colorectal Cancer. Future Oncol (2019) 15(22):2571-6. doi: 10.2217/fon2018-0454 consent to participate in this study was provided by the participants' legal guardian/next of kin. Written informed consent was obtained from the individual(s) for the publication of any potentially identifiable images or data included in this article.

\section{AUTHOR CONTRIBUTIONS}

Conceptualization: SZ. Project administration: YS. Writing original draft: YS. Writing - review and editing: SZ. All authors contributed to the article and approved the submitted version

6. Li J, Qin S, Xu RH, Shen L, Xu J, Bai Y, et al. Effect of Fruquintinib vs Placebo on Overall Survival in Patients With Previously Treated Metastatic Colorectal Cancer: The FRESCO Randomized Clinical Trial. Drugs (2018) 78(16):175761. doi: 10.1007/s40265-018-0998-Z

7. Shirley M. Fruquintinib: First Global Approval. Drugs (2018) 78(16):1757-61. doi: 10.1007/s40265-018-0998-z

8. Chen J, Wang J, Lin H, Peng Y. Comparison of Regorafenib, Fruquintinib, and TAS-102 in Previously Treated Patients With Metastatic Colorectal Cancer: A Systematic Review and Network Meta-Analysis of Five Clinical Trials. Med Sci Monit (2019) 25:9179-91. doi: 10.12659/MSM.918411

Conflict of Interest: The authors declare that the research was conducted in the absence of any commercial or financial relationships that could be construed as a potential conflict of interest.

Copyright (c) 2021 Shu and Zheng. This is an open-access article distributed under the terms of the Creative Commons Attribution License (CC BY). The use, distribution or reproduction in other forums is permitted, provided the original author(s) and the copyright owner(s) are credited and that the original publication in this journal is cited, in accordance with accepted academic practice. No use, distribution or reproduction is permitted which does not comply with these terms. 